\title{
Does environmental reporting matter?
}

\begin{abstract}
International media attention and increased public concerns have created global environmental issues, thus, companies now face great pressure to engage in environmental activities while environmental reporting (ER) acts as a medium for disclosure of their environmental responsibility in fulfilling the social needs. Therefore, embarking on effective environmental engagement will sustain better life. Thus, this study purposes to evaluate the impacts of ER practices on financial performance using the outcome indicators of return on equity (ROE), return on assets (ROA) and earnings per share (EPS). Furthermore, this study also makes comparison between companies in ESI and non-ESI. This study has employed the stratified random sampling technique in order to determine the sample size from the population of ESI and non-ESI companies. Content analysis was adopted to examine the annual reports of 78 Malaysian PLCs from years 2010 until 2014 through the usage of measurement of volume in terms of sentences and the disclosure index to determine the quantity of ER and quality of ER respectively. The analysis shows that there is no significant relationship between ER practices and ROE and ROA, whereas there is a significant positive relationship between the existence of ER and EPS. For ESI companies, there is significant positive relationship between existence of ER and financial performance while a significant negative relationship is found between quality of ER and financial performance. On the other hand, there is no evidence of significant relationship to be found between ER practices and financial performance of non-ESI companies. Furthermore, it can be clearly seen that the existence of ER is higher in ESI companies as compared to nonESI companies. This research has shown that the number of disclosing companies is promising, however the quantity and quality of ER is still unfavorable where the PLCs are still lacking in providing environmental information needed by the public. Nevertheless, since ER is still a voluntary move in Malaysia, regulators are advised to take initiatives to enhancing this practice through communicating the benefits to the companies and at the same time ensure the mandatory adoption of the ER practices in terms of the quantity and quality.
\end{abstract}

Keyword: Environmental reporting practices; Financial performance; Public listed companies; Environmentally sensitive industries; Non-environmentally sensitive industries; Malaysia 
